\title{
MODAL SURVEY TEST OF THE SOTV 2 X 3 METER OFF-AXIS INFLATABLE CONCENTRATOR
}

\author{
Robert C. Engberg* \\ John O. Lassiter** \\ Structures, Mechanics, and Thermal Department \\ NASA/Marshall Space Flight Center \\ Huntsville, AL 35812 \\ Jennie K. McGee \\ SRS Technologies \\ Systems Technology Group \\ Huntsville, AL 35806
}

\begin{abstract}
NASA's Marshall Space Flight Center has had several projects involving inflatable space structures. Projects in solar thermal propulsion have had the most involvement, primarily inflatable concentrators. A flight project called Shooting Star Experiment initiated the first detailed design, analysis and testing effort involving an inflatable concentrator that supported a Fresnel lens. The lens was to concentrate the sun's rays to provide an extremely large heat transfer for an experimental solar propulsion engine. Since the conclusion of this experiment, research and development activities for solar propulsion at Marshall Space Flight Center have continued both in the solar propulsion engine technology as well as inflatable space structures. Experience gained in conducting modal survey tests of inflatable structures for the Shooting Star Experiment has been used by dynamic
\end{abstract}

\footnotetext{
*Aerospace Technologist, Structural Dynamics; Member AIAA

** Aerospace Technologist, Structural Dynamics; Senior Member AIAA

-Associate Engineer, Aerospace Technologies Directorate

"Copyright (C) 1999 by the American Institute of Aeronautics and Astronautics, Inc. No Copyright is asserted in the United States under Title 17, U.S. Code The US Government has a royalty-free license to exercise all rights under the copyright claimed herein for Governmental purposes. All other rights are reserved by the copyright owner."
}

test engineers at Marshall Space Flight Center to conduct a modal survey test on a Solar Orbital Transfer Vehicle (SOTV) off-axis inflatable concentrator. This paper describes how both previously learned test methods and new test methods that address the unique test requirements for inflatable structures were used. Effects of the inherent nonlinear response of the inflatable concentrator on test methods and test results are noted as well. Nine analytical mode shapes were successfully correlated to test mode shapes. The paper concludes with several "lessons learned" applicable to future dynamics testing and shows how Marshall Space Flight Center has utilized traditional and new methods for modal survey testing of inflatable space structures.

\section{INTRODUCTION}

For the last three years, NASA's Marshall Space Flight Center has had projects that involve inflatable space structures. A solar thermal propulsion project has had the most involvement with inflatable space structures, primarily concentrator assemblies. A flight project called Shooting Star Experiment initiated the first detailed design, analysis and testing effort involving an inflatable concentrator. The concentrator had a torus that supported a Fresnel lens. This lens was designed to concentrate sun light and focus it on the combustion chamber of an experimental solar engine.

The Structures, Mechanics, and Thermal Department at Marshall Space Flight Center has conducted static, dynamic, and thermal tests in both ambient and vacuum conditions on inflatable subcomponents such as struts and for several designs of a full scale inflatable concentrator. Modal survey tests were performed on struts and inflatable concentrators. Strut static stiffness tests and an inflatable concentrator thermal test in vacuum conditions were performed. The latter test, called a terminator test, simulated the quasi-static response the concentrator would have due to the transition from darkness to sun light while in orbit. Inflation and deployment tests were also conducted. ${ }^{1.2 .3}$ 
The organizations that were involved in design, analysis, and testing of inflatable concentrators for the Shooting Star Experiment have continued their work for the Marshall Space Flight Center program office that managed the experiment. Their goal was to further advance the capability to analytically model the structural dynamics of thin film inflatable concentrators and to conduct the dynamic tests that are to be used to verify the analyses. These follow-on tests have allowed the team to gain additional experience in using test hardware and test methodologies that are uniquely suited for the dynamic testing of these light weight structures.

As a means of capitalizing on their past experience as well as to gain additional experience, the Modal and Control Dynamics Team of the Structural and Dynamics Test Group was asked to conduct a modal survey test of an inflatable concentrator developed by SRS

Technologies. Modal parameters from the test validated a finite element model that will be used for concentrator pointing and control analyses.

The test article used for these modal survey tests was developed for use in a solar thermal propulsion system. Solar thermal propulsion is the advanced upper stage propulsion system option currently being developed for use with the Air Force's Solar Orbital Transfer Vehicle (SOTV) Program. This vehicle can be used for several modes of operation including propulsion, communications/surveillance, and power generation. The SOTV design has dual opposing large area deployable collectors that focus the Sun's energy into an absorber which converts the solar energy into electrical power and rocket propulsion. In the propulsion mode, hydrogen gas is heated and then expands through a nozzle, thereby providing thrust to transfer the vehicle from low earth orbit to geosynchronous orbit. In the power mode, thermionic diodes are used to convert heat into electricity. Figure I is a drawing of the SOTV.

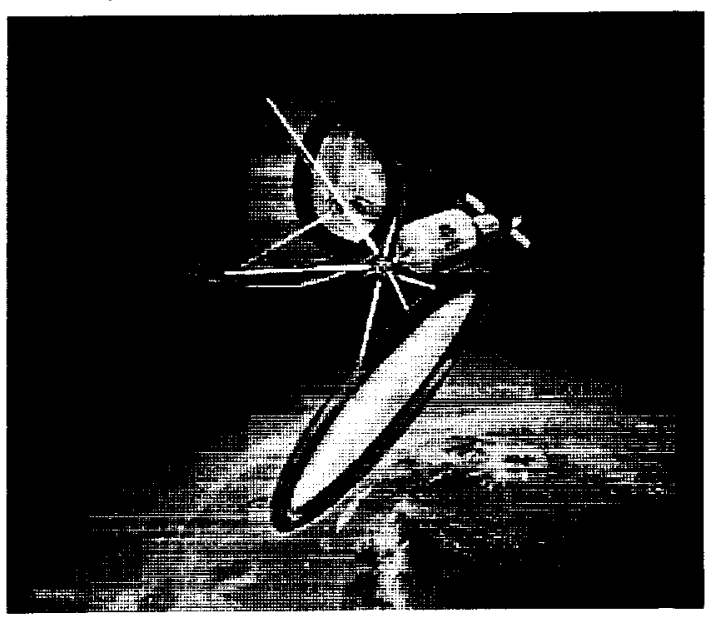

Figure 1 Artist Caption of the Solar Orbital Transfer Vehicle

\section{THE TSC-6 2 X 3 METER OFF-AXIS CONCENTRATOR}

Highly accurate solar concentrators are required to meet the power requirements of SOTV. SRS Technologies developed a Torus- $\underline{\text { Supported }}$ Concentrator called TSC -6 under a NASA/Glenn Research Center (GRC) contract to demonstrate the feasibility of designing and fabricating large inflatable solar concentrators to meet the design requirements of space solar dynamic systems such as the SOTV. The Air Force has tested TSC-6, seen in Figure 2 below, as a candidate SOTV concentrator design.

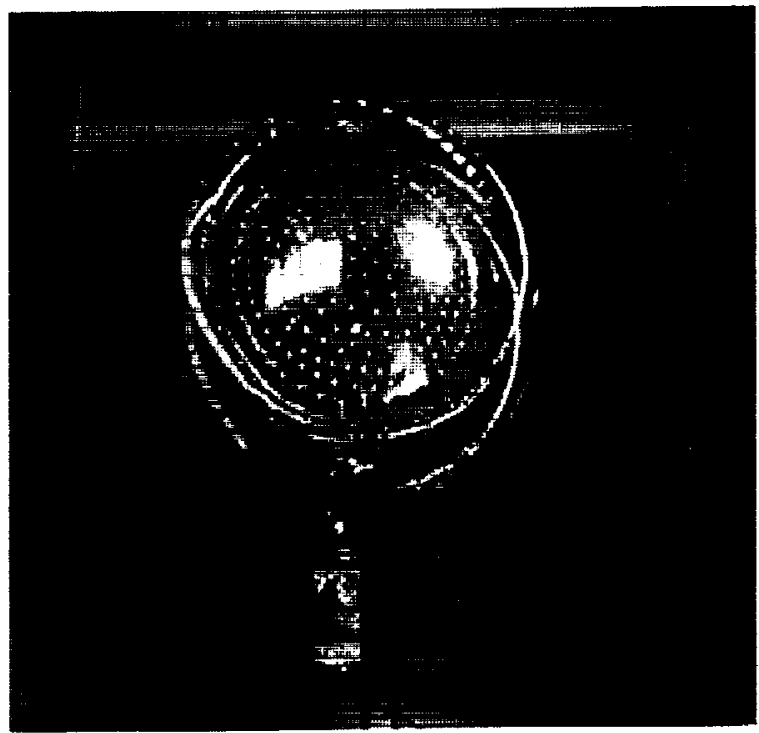

Figure 2. The TSC-6 Off-Axis Inflatable Concentrator 
TSC- 6 is a 2 meter by 3 meter ( 6.60 feet by 9.90 feet) class inflatable concentrator consisting of a lenticular, torus, catenary system, and support struts. The inflated lenticular consists of two attached .001 inch polyimide films, a canopy and a reflector film, made from CP1 polyimide licensed by the NASA Langley Research Center and produced at SRS Technologies. These parabolic shaped films are designed and fabricated at SRS Technologies to address NASA's system requirements. The inflated elliptical torus consists of .0005 inch Kapton film. A catenary system consisting of 92 lengths of elastic silicon tubing joins the torus and lenticular. Three composite struts with a wall thickness of 0.030 inches support the concentrator. Figure 3 shows the dimensions of the TSC- 6 .
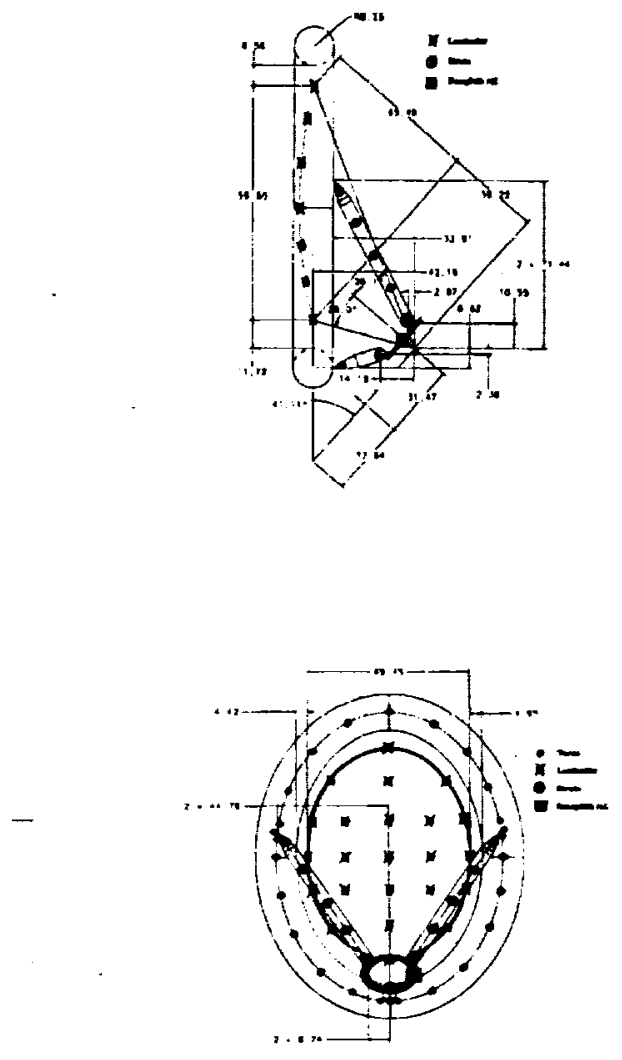

Figure 3. TSC-6 Dimensions

\section{PREVIOUS ANALYSES AND TESTS OF THE TSC-6 CONCENTRATOR}

In an effort to design a large inflatable concentrator that meets solar dynamic system requirements, the Air Force, NASA GRC, Thiokol Propulsion, and SRS Technologies collaborated to test and analyze the TSC-
6 inflatable concentrator. This effort included finite element modeling, thermal modeling, photogrammetry, thermal vacuum testing, geometric ray trace modeling, on-sun testing, and deployment testing.

ALGOR nonlinear finite element analysis software was used to create a model of the TSC-6 (Figure 4) to predict and optimize its performance. Photogrammetry was used to characterize the shape change of the reflector between two pressure configurations and the results were compared to the shape change of the finite element model analyzed at two pressure configurations. It was found that the change in shape was virtually identical between the TSC- 6 model and the hardware and therefore it was verified that the model accurately represents the response of TSC- 6 . Modeling techniques used on TSC- 6 have been used to create models of larger inflatable concentrators and to optimize these models to null out any undesirable deflections caused by thermal and pressure loads such that the slope error of the concentrator falls within the design requirements.

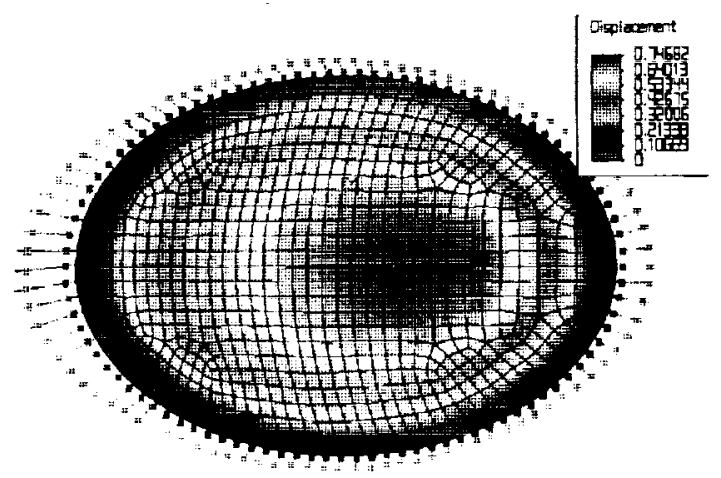

Figure 4. TSC-6 Finite Element Model

Thermal models of TSC- 6 were created in order to understand the thermal environments and environmental effects on inflatable concentrators. These models were generated using TRASYS (Thermal Radiation Analyzer System) and SINDA (Systems Improved Numerical Differencing Analyzer). Information resulting from these models included radiation conductors, surface heat flux, and nodal temperatures of the concentrator. In order to verify the accuracy of these SINDA and TRASYS models, TSC- 6 was tested in a NASA thermal vacuum facility and a thermal model was created. The model results were compared to the test results and verified that the thermal models accurately predict thermal effects on inflatable structures.

In addition to the thermal and structural modeling, geometric ray trace models were created and deployment tests were performed on TSC-6. Geometric 
ray trace models were generated using OptiCAD geometric ray trace software to characterize the spot size and flux distribution of the concentrator. The results of these models were verified through on-sun testing of TSC-6. Tests were also performed to demonstrate the deployment of large inflatable concentrators in ambient and vacuum environments. Results of these hardware tests along with thermal, structural, and optical modeling, have been valuable to the process of designing a large inflatable concentrator that meets the requirements of solar dynamic systems. Modal survey tests such as those described in this paper will also aid in the design and optimization of inflatable concentrators.

\section{DESCRIPTION OF THE MODAL TEST SETLP}

The fixed base modal survey test was performed in a SRS Technologies high bay facility. Figure 5 shows the concentrator and the general arrangement of the test equipment. The ends of each of the three composite struts opposite from the concentrator were attached to a metal support ring via an arrangement of brackets and turn buckles. The support ring was bolted at four locations to the floor of the high bay. In order to facilitate the rotation of the concentrator for response point measurements, these four locations were $90^{\circ}$ apart.

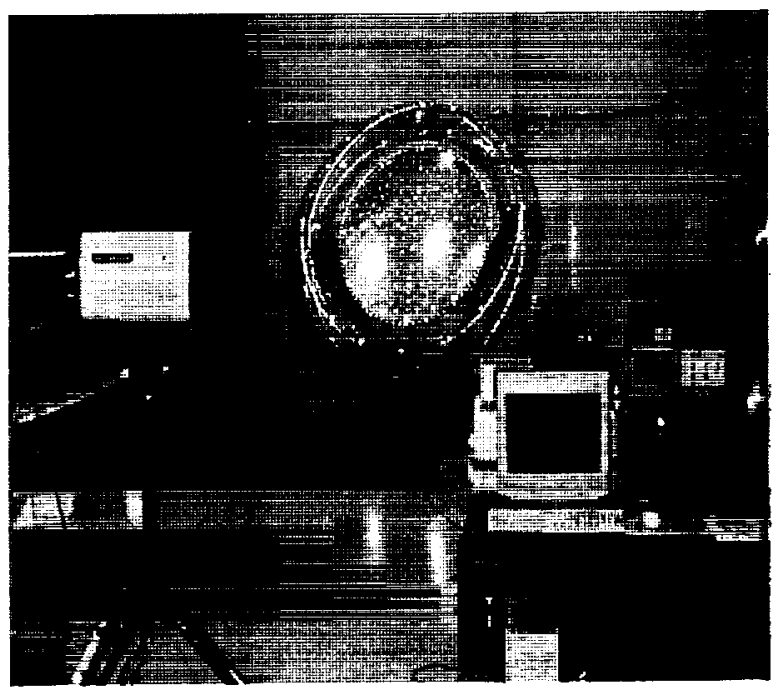

Figure 5. TSC-6 Modal Survey Test Setup

$\therefore$ ingle pressurization system was used to supply two separate liness to the concentrator and the lenticular. For the modal survey test, mode shapes were identified for two wrus lenticular pressure configurations. Contiguration 1 had a torus pressure of $0.072 \mathrm{psi}$ and a lenticular pressure of $0.000795 \mathrm{psi}$. Configuration 2 had the same torus pressure as Configuration 1 but with a lenticular pressure of $0.00065 \mathrm{psi}$.

During testing, a fault in the pressurization system caused excess pressurization of the torus. This caused a tear in the top section of the torus that was immediately repaired. A few of the FRF's that were calculated before the tear were recalculated after repairs were made. A comparison of these FRF's to those calculated before the tear showed no significant differences and a conclusion was made the repaired tear did not cause any changes to the concentrator's dynamic response.

For the most part, the test hardware and procedures utilized for this inflatable concentrator modal survey test are the same used for previous inflatable concentrator tests. However, since the TSC- 6 has components that are not inflated (the composite struts) and inflated components, the instrumentation arrangement used for response point measurements is different. Also, several different post processing procedures not used in previous tests were employed after measurement of frequency response functions (FRF's). These procedures will be discussed later in the paper. Strut responses were measured using triaxial accelerometers. Mass loading of the composite struts by the accelerometers was considered negligible.

Torus and lenticular response measurements were made using an Ometron ${ }^{\mathrm{TM}}$ scanning laser vibrometer. Like several previous tests of inflatable concentrators conducted at Marshall Space Flight Center, the primary reason for employment of a noncontacting measuring system such as the vibrometer is to prevent mass loading of the test article. Vibrometer test procedures used for this test were developed during the course of conducting earlier modal survey tests on other inflatable concentrators and their components. The most notable similarity was the use of small sections of retroreflective tape as surface preparations for torus and lenticular response points and on comer cubes at the torus response points. The surface preparation on the torus and the back side of the lenticular was needed since these surfaces were transparent and would allow the laser to go through. This would result in a less than adequate strength of the retum laser signal and would degrade the velocity measurements. The front side of the lenticular had a reflective surface, but small sections of reflective tape were used at each response point to not only improve the strength of the return laser signal but also to locate and identify the response point locations.

The comer cubes seen in Figure 6 were placed on the top of the torus. They were used for making measurements on the torus in the $\mathrm{Y}$ axis. As seen in 
Figures 3 , the top and bottom of the torus are perpendicular to the $Z$ axis of the concentrator. Their use allowed the laser to be perpendicular to a surface at all locations. Without use of the comer cubes, torus

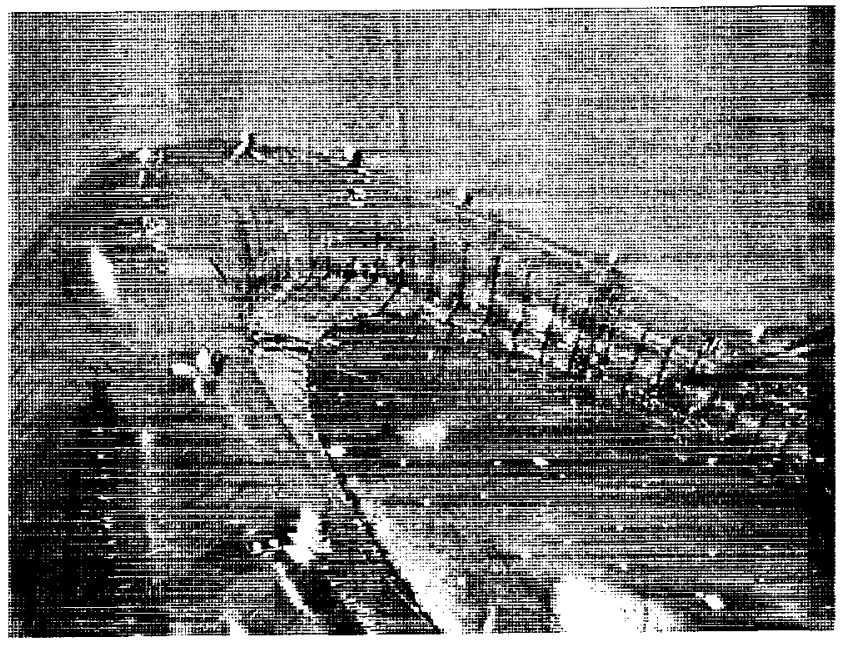

Figure 6. Comer Cubes at Torus Response Points

response points would have to be on the side of the torus as viewed in the $Y$ axis. Also, the laser would be at acute angles to the torus surface for those response points located at the upper and lower ends (positive $\mathrm{X}$ and negative $\mathrm{X}$ directions respectively) causing poor velocity measurements. Any mass loading and stiffness affects on the torus from the comer cubes were considered negligible.

All 67 response points used in this test are seen in Figure 3. Inspection of these figures reveals an aspect that. was not encountered in any other inflatable concentrator modal survey test prior to this one. The torus and lenticular, both top and bottom surfaces, are very close to each other. This proximity added some difficulty in viewing and interpreting the mode shapes.

Another procedure used from a previous test is rotating the inflatable concentrator to make velocity measurements with the vibrometer in as many of the three axes as possible and at many points as possible. Rotation was first used in a test where a concentrator was placed in a thermal-vacuum chamber for response point measurements at vacuum and cryogenic temperatures. Since the vibrometer had to be positioned outside the chamber at the only port hole available, it could not be moved to make measurements in all orthogonal axes. In this particular test, $\mathrm{Z}$ axis measurements could not be made. However, after $\mathrm{X}$ axis measurements were made for all test conditions, the concentrator was rotated $90^{\circ}$ so that $Y$ axis measurements could be made. For the TSC- 6 test, there were for the most part no restrictions in viewing the concentrator along any local axis or global axis. However, in order to keep the vibrometer positioned in one specific area, $\mathrm{X}$ and $\mathrm{Z}$ axis measurements for the torus and lenticular were measured first. Then the concentrator was rotated $90^{\circ}$ so that $Y$ axis measurements on the torus could be made. Due to possible mass loading of the reflective side of the lenticular and the obstructed view of the lenticular by the torus in the $Y$ direction, corner cubes were not employed; therefore $\mathrm{Y}$ axis measurements on the lenticular were not made. For measurements on the back side of the lenticular as well as points located near the strut/torus interfaces, the concentrator was rotated $90^{\circ}$ for the second time.

In the report for the concentrator tested in the thermalvacuum chamber and corresponding technical paper ${ }^{2,7}$, the errors encountered in the measurements of FRF's due to the inability of placing the vibrometer laser beam directly in line with an axis of the concentrator are discussed in detail. These errors, called "cosine theta" errors, are caused by the angles the laser beam (also the velocity vector being measured) makes with each of the three orthogonal axes of the concentrator coordinate system when placed at a response point. For the above referenced test, the distance between the concentrator and the vibrometer was determined to be large enough so that the angles between the laser beam (velocity vector) and the test article's $X$ and $Y$ axes were small. This allowed $X$ and $Y$ axis vibrometer measurements to be considered directly along these axes and eliminated the need for the resolution of the resulting FRF's into components.

For the TSC- 6 test, FRF's calculated using velocity measurements in the global $\mathrm{X}$ axis (parallel to the high bay floor) were resolved into local (inflatable concentrator) $\mathrm{X}$ and $\mathrm{Z}$ axes by multiplying these global FRF measurements by the respective sine or cosine of the angle the laser beam made with the $X-Y$ plane of the tonus (Figure3). The actual resolution was accomplished by multiplying the global FRF's by the scalar value of the sine or cosine of the angle. This was accomplished by using the math function of the data acquisition software. To obtain the complete local $X$ and $Z$ torus and lenticular responses, global $Z$ axis velocity measurements on the torus and lenticular also should have been made. Their resulting FRF's, once resolved into $X$ and $Z$ axis components, would be added to the local $X$ and $Z$ FRF's made from the global $X$ axis However, the vibrometer could not be readily placed directly above or below the concentrator to make global $\mathrm{Z}$ axis velocity measurements.

As previously mentioned, a post FRF measurement process was needed that was not required in earlier 
tests. The mixture of accelerometers for strut response measurements and the laser vibrometer for torus and lenticular measurements resulted in both inertance and mobility FRF's being calculated. Since most of the response points were on the inflated components where the laser vibrometer was used, it was decided that the inertance FRF's calculated using the accelerometer measurements would be converted to mobility FRF's. Mathematically this is accomplished by integrating the inertance FRF's. The integration was accomplished by dividing the inertance FRF's by $j \omega$ using the math function of the data acquisition software. Curve fitting for modal parameter identification requires all FRF's to be the same type.

Test hardware and setup for the TSC- 6 modal survey test are similar to previous tests and will be briefly described. Single point random excitation was provided by a Ling TM four pound force nominal electrodynamic shaker located at the middle of one of the two longer struts. A Hewlett-Packard ${ }^{\mathrm{TM}}$ source module provided the random signal to the shaker. For FRF acquisition, the source signal was filtered from 0.0 to $100.0 \mathrm{~Hz}$ with a high-low pass filter. Force input was measured by a load cell with a $500 \mathrm{mV} / \mathrm{lbf}$ nominal voltage sensitivity. Both force, acceleration and velocity measurements were acquired with a Hewlett-Packard ${ }^{\text {TM }} 3566 / 67$ Dynamic Signal Analyzer (DSA) with the acquisition software residing on a laptop computer. All accelerometer outputs were simultaneously acquired as one set of data. Velocity measurements were obtained one response point at a time by scanning the vibrometer to each point.

FRF calculation was performed by the acquisition software that was set up to take 25 averages over a bandwidth of 0.0 to $50 \mathrm{~Hz}$ with a resolution of 0.125 $\mathrm{Hz}$. The Hanning window was used to minimize the effects of leakage. As previously discussed, conversion from inertance to mobility FRF's and the "cosine theta" error corrections were performed using the DSA software. Figure 7 is one mobility FRF that were calculated. Characteristics seen in these FRF's such as high damping and close mode shape coupling in some parts of the bandwidth are similar to those obtained in previous modal survey tests.

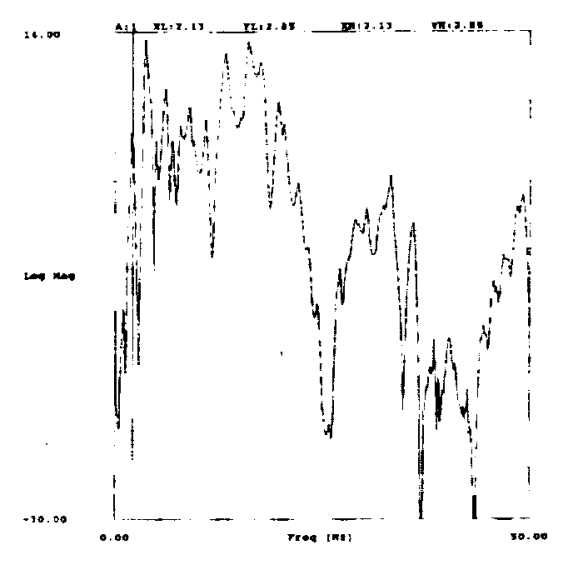

Figure 7. Mobility Frequency Response Function

\section{LINEARITY ASSESSMENT OF THE TSC-6 CONCENTRATOR}

Usually in modal survey tests of more typical structures (metallic, composite, or both), linearity checks using sinusoidal sweeps are conducted after FRF's are calculated and a set of experimental modal parameters are obtained. In most, if not all cases, the structure being tested shows linear response using forces levels similar to what was used to excite the structure during the modal survey test. Force levels used in modal survey tests are always substantially lower than any levels experienced while in use, in operation, etc. Also in most if not all cases the structure being tested responds linearly to an actual dynamic force environment. The linearity checks are primarily performed to identify any nonlinear response due to free-play in moving parts, lose joints, test article/fixture interface free-play, etc.

The light weight characteristic of an inflatable structure is the reason they are sensitive to mass loading affects. Closely associated with being light weight and flexible is the exhibition of nonlinear response at force levels significantly lower than traditional metallic/composite structures. For this and previous modal survey tests of inflatable concentrators, linearity checks were conducted prior to calculating FRF's. These linearity checks were used to determine force levels that produce a linear response for modal parameter identification and at what level nonlinear response starts.

At the beginning of the TSC- 6 test, three sinusoidal sweeps were performed at $0.125 \mathrm{lbf}, 0.250 \mathrm{lbf}$, and $0.375 \mathrm{lbf}$. The same electrodynamic shaker and input point used for obtaining FRF's for modal parameter 
identification were used for the sinusoidal sweeps. Due to the low frequency peak force level limitation of the shaker, the sinusoidal source signal from the DSA was filtered above $5.0 \mathrm{~Hz}$. FRF's using the response at the shaker input location (drive point) and FRF's using a response point on the torus were used in the linearity assessment.

Figures 8, 9, and 10 show the FRF's for each of the three sinusoidal force levels. The $0.25 \mathrm{lbf}$ level is used as a reference and is compared to the other two force levels. In Figure 8, the dashed trace is the $0.125 \mathrm{lbf}$ FRF and the solid trace is for the $0.250 \mathrm{lbf} F R F$. The $0.125 \mathrm{lbf}$ force level shows a resonant peak at $19.5 \mathrm{~Hz}$. Figure 9 is the same two FRF's overlaid on each other with a peak shown at $19.25 \mathrm{~Hz}$. Note also the peak in the $0.250 \mathrm{lbf} F R F$ that is above both of these peaks. The increase in force level from $0.125 \mathrm{lbf}$ to $0.250 \mathrm{lbf}$ has caused the $19.5 \mathrm{~Hz}$ peak to split into two peaks. This is a nonlinear response called bifurcation. Figure 10 has the $0.250 \mathrm{lbf} F R F$ as the solid trace and the $0.375 \mathrm{lbf}$ as the dashed trace. The effects of nonlinear response is further seen by the two peaks about $19.5 \mathrm{~Hz}$ being split further apart.

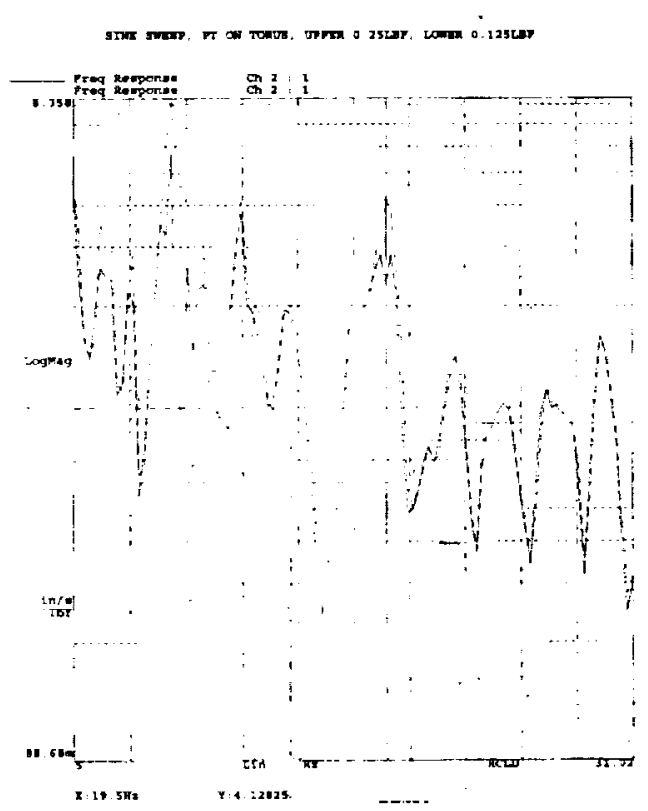

Figure 8. $0.125 \mathrm{lbf}$ and $0.250 \mathrm{lbf}$ Sinusoidal Sweep FRF

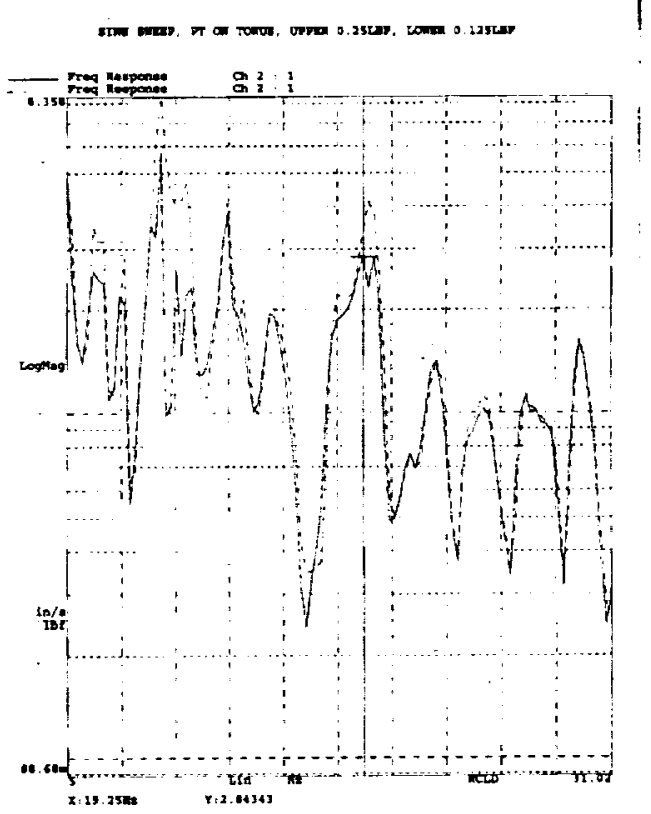

Figure 9. $0.125 \mathrm{lbf}$ and $0.250 \mathrm{lbf}$ Sinusoidal Sweep FRF with $19.25 \mathrm{~Hz}$ Peak Indicated

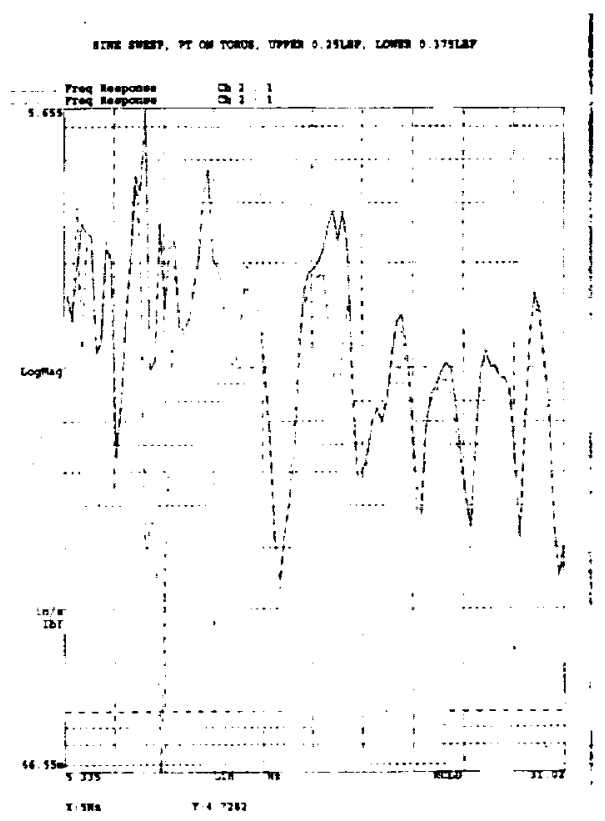

Figure 10. $0.375 \mathrm{lbf}$ and $0.250 \mathrm{lbf}$ Sinusoidal Sweep FRF

It is obvious that the above force levels produced a nonlinear response and therefore force levels for modal parameter identification should be lower. In this and previous tests, force levels were and have been significantly lower. Figures 11 and 12 show FRF's generated from RMS force levels of $24.3 \mathrm{mlbf}$ and 14.6 
mlbf respectively. By comparing similar regions of these two FRF's (shown by the arrows), it is seen that even these force levels can show a bifurcation in some part or parts of the frequency range not readily seen in the sinusoidal sweep FRF's. The emphasis on using a force level that produces linear response may be a mute point since the inflatable concentrator material (Kapton) has frequency dependent material properties that could result in periodically modulated response regardless of the force level used ${ }^{8}$. Still, an attempt was made to produce a linear response by keeping the RMS value of the random force levels at or below $14.6 \mathrm{mlbf}$. The response is then considered linear for this particular force level.

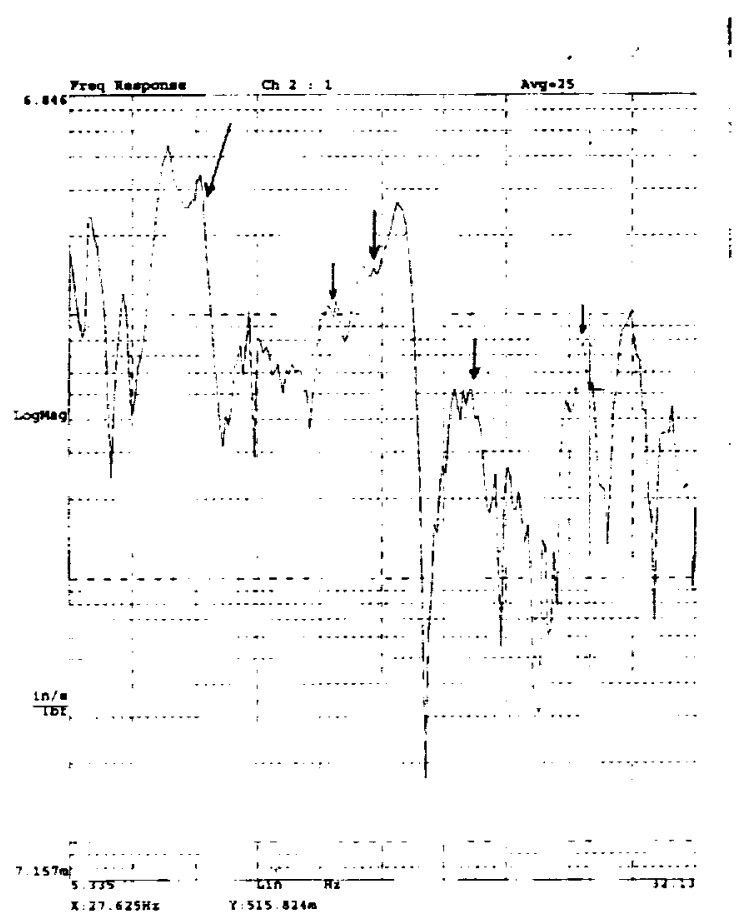

Figure 11. FRF for a $24.3 \mathrm{mlbf}$ RMS Force Level

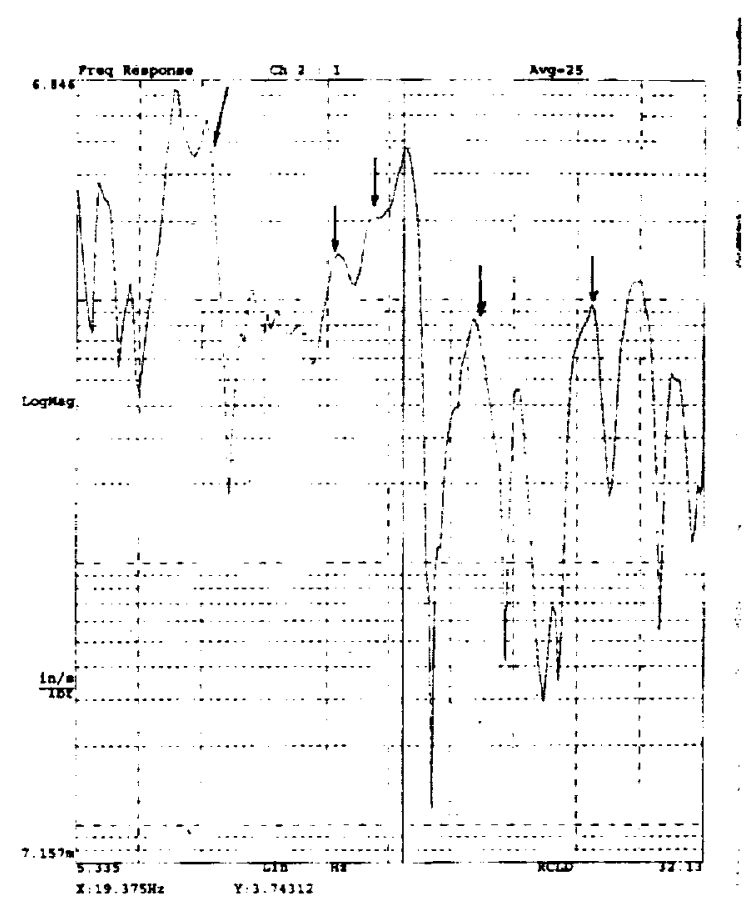

Figure 12. FRF for a $14.6 \mathrm{mlbf}$ RMS Force Level

\section{EXPERIMENTAL MODAL PARAMETER IDENTIFICATION}

As discussed in an earlier section, all of the FRF's calculated from both the measured acceleration and velocity responses had to be the same type before they could be used in any curve fitting routine. After the inertance FRF's using the acceleration measurements were converted to mobility FRF's, they were combined with the mobility FRF's calculated from the velocity measurements made with the laser vibrometer. Therefore a complete set of mobility FRF's for all 67 response points were available for modal parameter identification. The Spectral Dynamics StarStruct ${ }^{\mathrm{TM}}$ personal computer based software provided several curve fitting routines for use in identifying mode shapes with their frequencies and damping. Before the FRF's could be used, they were translated from the Standard Data Format to a format compatible to StarStruct ${ }^{\mathrm{TM}}$.

The Advanced Curve Fit routine available in StarStruct ${ }^{\mathrm{TM}}$ was used for all test cases. This routine calculates a Mode Indicator Function (MIF) and Stability Diagram using all of the FRF's. Only those poles in the Stability Diagram that were stable for most model orders and were coincident with predominant peaks on the MIF were selected as modal resonances. Prior to curve fitting, the Modal Peaks function in StarStruct ${ }^{\mathrm{TM}}$ was used to obtain an initial count of resonant peaks present in the FRF's. The model order 
for the Advanced Curve Fitting routine was selected to be twice this estimate of resonant peaks. With each pole selected to be a system resonant frequency a corresponding damping value was provided. Once this modal table was developed, it was used along with all FRF's to calculate the modal vectors that correspond with each frequency and damping value. Selection of independent (orthogonal) mode shapes was accomplished using a Modal Assurance Criteria (MAC) matrix. StarStruct ${ }^{\mathrm{TM}}$ has both mode shape animation and hard copy function using multiple views.

\section{DISCUSSION OF RESULTS}

Eighteen mode shapes were identified for the first pressure configuration and 16 for the second configuration. The values of the off diagonal elements of the MAC matrix for each set of mode shapes were used to determine the degree of orthogonality of the mode shapes. Usually if a mode shape has off diagonals of 0.1 or lower, that mode shape is considered independent of the other mode shapes and is therefore a mode shape of the system. In previous modal survey tests on inflatable concentrators, several mode shapes that were identified had off diagonals well above 0.1 . For modal surveys of more traditional structures, large off diagonals are most likely the result of a lack of spatial resolution, or, in other words, there is not a sufficient number of response points to be able to separate from a vector standpoint one mode shape from another. However with characteristics such as high damping, nonlinear response, difficulty in obtaining a sufficient energy distribution to excite all modes of interest due to limitations in the location and type of excitation, off diagonals higher than 0.1 were considered to be acceptable. For a previous test of an inflatable concentrator, all mode shapes with off diagonals of 0.4 or lower were considered to be orthogonal to each other and were selected as independent mode shapes.

Most of the mode shapes identified in this test had off diagonals less than 0.1 ; in fact this was the first test where most of the mode shapes identified using the StarStruct ${ }^{\mathrm{TM}}$ had off diagonals this low. One possible explanation is the TSC- 6 test had fixed-free boundary conditions. All previous tests were conducted with free-free boundary conditions. Fixed-free boundary conditions plus the ability to place the excitation source location directly on a major component of the structure that is not inflated provided a better excitation and therefore better energy distribution into the structure. Therefore, all mode shapes of interest were better excited than in previous tests. Those mode shapes with off diagonals above 0.4 were selected only if their corresponding roots showed strong stability and were aligned with a dominant peak of the MIF. For these cases, lack of spatial resolution is considered to be the only reason for the high off-diagonals.

Figures 13 through 16 are two views of the third mode shape for each pressure configuration. The proximity of the response points on both sides of the lenticular to those on top of the torus make it somewhat difficult to determine relative motion, in particular when viewing the animated mode shapes. Combinations of torus bending and twist about its major and minor axes, lenticular bending and twist, and lenticular $Z$ axis translation can be seen in the lower order modes (i.e., modes 1 through 7) especially when viewing directly in the $\mathrm{Y}$ axis. This type of response is expected for lower order global modes of a structure with these geometric characteristics. Like any structure, the higher order mode shapes are more difficult to define. In comparing mode shapes of the two configurations, identical response of both the torus and lenticular is seen, especially the torus. Some differences are seen in the lenticular and this is attributed to the difference in lenticular pressure.

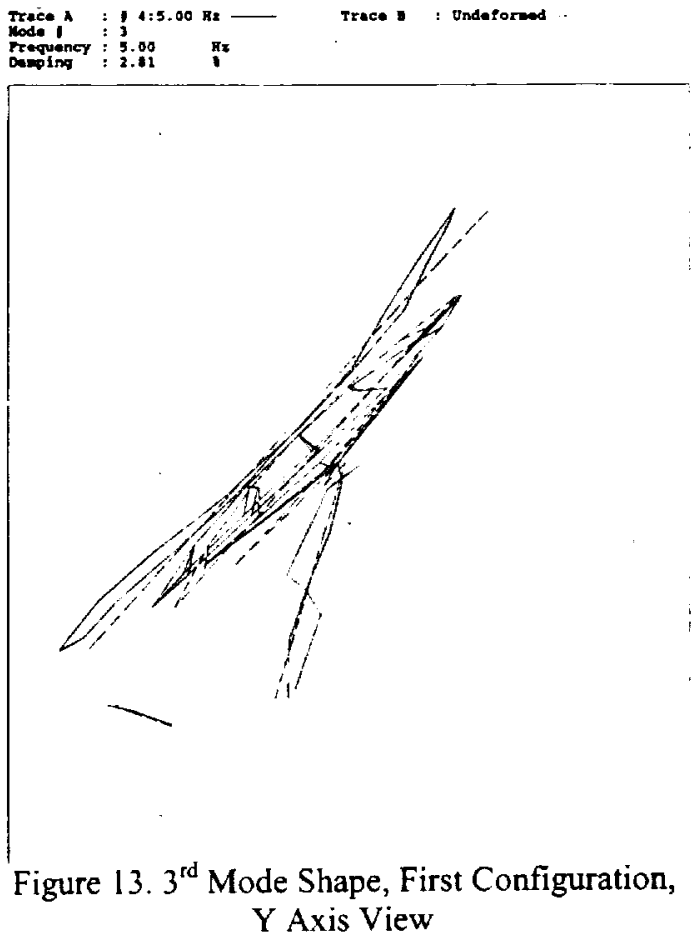




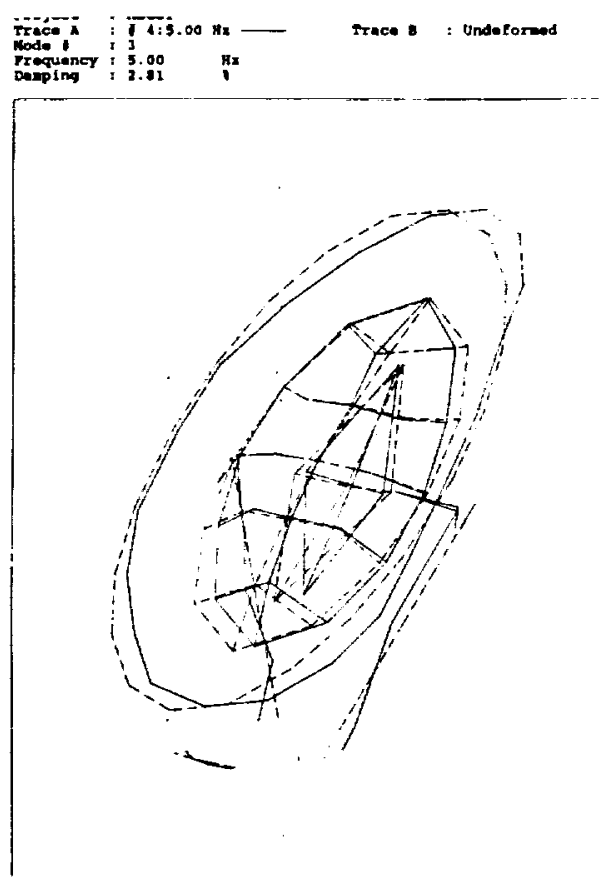

Figure 14. $3^{\text {rd }}$ Mode Shape, First Configuration, Orthogonal View

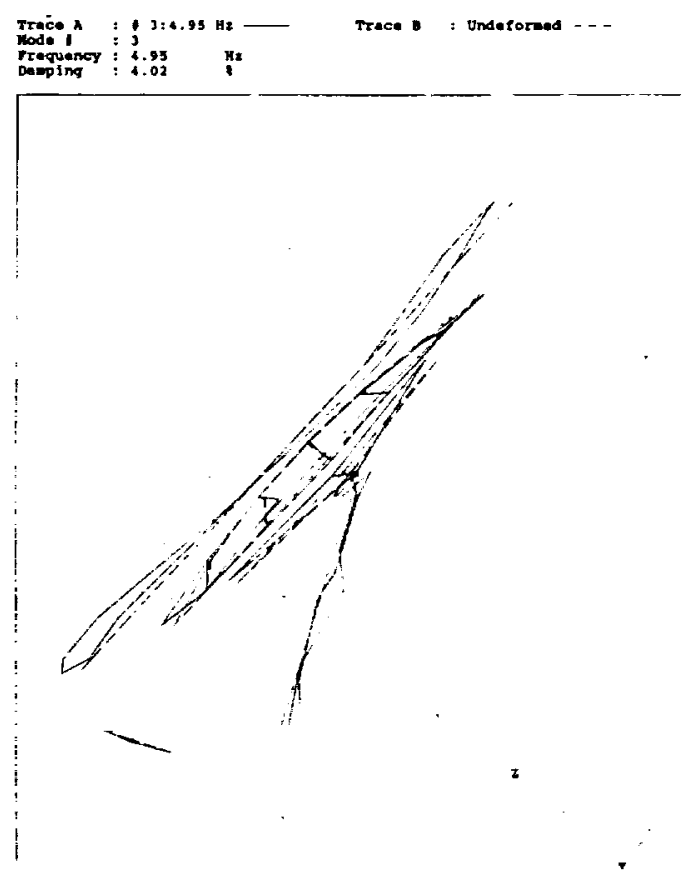

Figure $15.3^{\text {rd }}$ Mode Shape, Second Configuration, $Y$ Axis View

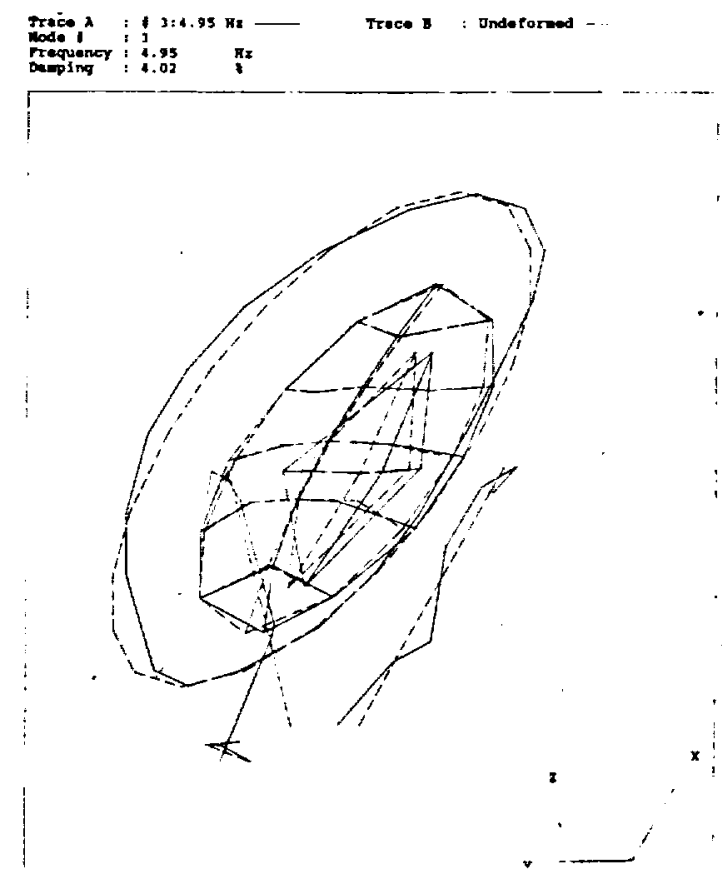

Figure 16. $3^{\text {rd }}$ Mode Shape, Second Configuration, Orthogonal View

Damping values for both configurations are similar to other inflatable concentrators. Damping for the first modes of both configurations are above $10 \%$. Damping decreases as modal order increases. Values for the highest order mode shapes are still well above 1 $\%$.

Response of the struts in both sets of mode shapes require additional descriptions. Motion at the response points closest to the support ring for the two longer struts is greater than expected and does not represent a fixed boundary condition. The response points at the ends of the two long struts were located about two inches above the hardware used to connect the struts to the support ring and were on the thin wall of the composite struts. Free play in the hardware (brackets, tumbuckles, etc.) used to connect the struts to the support collar and the flexibility of the strut at the lower response points is thought to be the cause of the higher than anticipated response. Response at these response points reduces as modal order increases. This is another indication of free play. The possibility of strut shell modes being excited was also considered to cause the large response; however, a separate strut analysis showed these modes to be above $70 \mathrm{~Hz}$.

After the modal survey test was completed, the two sets of modal parameters were used in validating a NASTRAN finite element model. The model uses 
tubular beam elements for the torus, struts, and catenaries. Quadrilateral shell elements are used for the lenticular. The model is compatible with the ADAMS software that will be used for control system dynamic analyses. To insure compatibility with the ADAMS software, nonlinear properties were not incorporated in the NASTRAN model ${ }^{9}$.

Initial comparison between test and analytical mode shapes showed the need for changes to the model. In order to simulate the free play in the hardware used to connect the struts to the support ring, some constraints in translation and rotation degrees-of-freedoms were removed. The swivel joint used to connect the tops of the struts to the torus was found to affect the dynamics enough to require them to be modeled as frictionless pivots. Also material properties of the thin film materials and composites had to be changed. Pressure in the torus and lenticular was modeled by adding to the modulus of elasticity in bending for the beam elements. Changes to the modulus of elasticity in bending were also used to take into account the wrinkling and localized buckling that was visually seen in the torus.

Since nonlinearities could not be included in the NASTRAN model in order to insure compatibility with the ADAMS software, not all of the mode shapes of the concentrator could be captured analytically.

Correlation between the model and test was performed by comparing frequencies and visually comparing mode shapes. In Table 1 it is seen that nine mode shapes from the model were correlated to test mode shapes for the second pressure configuration. These nine mode shapes are enough to provide a good identification of the inflatable concentrator system dynamics. For six of these mode shapes, the difference in frequency is below $10 \%$ and two are between $10 \%$ and $20 \%$. Model mode shapes above 14 were highly coupled and could not be found with a linear solution.

\begin{tabular}{|c|c|c|c|}
\hline Mode No. & Test & Model & $\%$ Error \\
\hline 1 & 2.06 & 2.03 & 1.50 \\
\hline 2 & 3.71 & 2.32 & 60.00 \\
\hline 3 & 5.00 & 5.61 & 1.10 \\
\hline 7 & 11.03 & 13.96 & 20.00 \\
\hline 8 & 13.43 & 14.63 & 8.00 \\
\hline 9 & 15.07 & 15.78 & 4.40 \\
\hline 12 & 19.63 & 19.07 & 2.90 \\
\hline 13 & 22.59 & 20.65 & 9.40 \\
\hline 14 & 24.80 & 21.88 & 13.30 \\
\hline
\end{tabular}

Table 1. Model to Test Frequency Comparison

\section{CONCLUSIONS}

The TSC- 6 modal survey test shows how Marshall Space Flight Center and the organizations it supports have developed the capability to utilize traditional and new methods for conducting modal survey tests of inflatable space structures. Even though the nonlinear dynamic characteristics of inflatable structures have added significant differences and difficulties not encountered in the testing of traditional space structures, the test successfully identified a set of experimental modal parameters for use in validating a dynamic analytical model.

In summary, the TSC- 6 modal survey test and the previous tests conducted at Marshall Space Flight Center have resulted in several "lessons leamed" that will benefit future structural tests of inflatable space structures. They are presented as follows:

1. The TSC-6 inflatable concentrator is an example where the test article has inflated and noninflated components. Having both of these distinct types of structural components required unique test instrumentation and data processing requirements not seen in typical modal survey tests. These requirements should be expected for future tests.

2. Special attention is required to insure the desired fixed or free boundary conditions are obtained and more than the usual amount of effort should be expended in order to obtain a set of desired boundary conditions. Any unknowns in boundary conditions further adds to the complexity of testing a structure with other more difficult characteristics (i.e. nonlinear response).

3. The use of electrodynamic shakers in the excitation of inflatable structures requires additional attention to the effects of shaker stingers. Stingers that are thinner and more flexible than those used in more typical modal survey tests will reduce or eliminate side loads being input into the inflatable and will help protect the inflatable from large, inadvertent excitations. When the only excitation point is located on an inflatable subcomponent (torus, strut, etc.), additional effort must be expended to determine which excitation method is better; either direct impact with an impulse hammer should be used or a shaker. Most likely, use of a shaker directly on a inflated component will not be practical due to localized flexibilities causing poor input force measurements.

4. Excitation of an inflatable structure can occur due to random acoustic or structural bome 
sources such as large pumps, air handlers, personnel activity, etc. The TSC- 6 test and another test encountered these random excitations that can be avoided by turning off all unnecessary machinery, testing when personnel activity is a minimum, etc.

5. Mass and stiffness of pressure system umbilicals have to be recognized, especially when correlating a model to test data. In the TSC-6 test, visual response of the umbilicals was seen when the concentrator was excited. Umbilical stiffness should be reduced as much as possible.

6. Special attention should be given to the operation of the pressurization system used for inflatable structures test. Procedures should be developed and utilized to prevent an excessive pressurization of the inflatable structure. Repair and test procedures, such as comparison of FRF's before and after excessive pressurization to assess changes to the inflatable structure's dynamic response, should be developed before testing begins.

\section{ACKNOWLEDGEMENTS}

The authors wish to thank Jim Moore and Randy Schauer of SRS Technologies for their support in this test.

\section{REFERENCES}

1. Engberg, Robert, and Lassiter, John, "Shooting Star Experiment, Pathfinder 2, Inflatable Concentrator Modal Survey in Vacuum Conditions", Dynamics Test Branch, Marshall Space Flight Center Test Report \# SSE-DEVED97-120, March, 1998.

2. Engberg, Robert, and Lassiter, John, "Shooting Star Experiment, Pathfinder 3, Inflatable Concentrator Modal Survey in Thermal-Vacuum Conditions", Dynamics Test Branch, Marshall Space Flight Center Test Report \# SSE-DEVED97-115, February, 1998.

3. Engberg, Robert, and Lassiter, John, "Shooting Star Experiment, Pathfinder 3, Inflatable Concentrator Terminator Test", Dynamics Test Branch, Marshall Space Flight Center Test Report \# SSE-DEV-ED98-046, July, 1998.
4. LMS International Memo, "Extraction of In-Plane Velocities using the CADA-X Laser Vibrometer Product", September, 1996.

5. Rumsey, Mark, et al, "In-Field Monitoring of Wind Turbines", Sound and Vibration, February, 1998, pp. 14-19.

6. Main, John A., et al, "Dynamic Analysis of Space-based Inflated Beam Structures", Journal of the Acoustical Society of America, February, 1995, pp. 1035-1045.

7. Engberg, Robert, and Lassiter, John, "Dynamic Testing of an Inflatable Structure under ThermalVacuum Conditions", $40^{\text {th }}$ AIAA Structures, Structural Dynamics, and Materials Conference, Paper No. 99-1519, St. Louis, MO, April, 1999.

8. Smith, Suzanne, et. al., "Nonlinear Interactions and the Hubble Space Telescope", AIAA, 1992.

9. Slade, Kara S., "Solar Orbital Transfer Vehicle (SOTV) Inflatable Concentrator Finite Element Analysis Report", Edmund T. Pratt, Jr. School of Engineering, Duke University, Durham, NC, December, 1999.

10. Engberg, Robert and Lassiter, John, "Solar Orbital Transfer Vehicle (SOTV) Inflatable Concentrator Modal Survey at SRS Technologies, Inc." Structural and Dynamics Test Group, Marshall Space Flight Center Test Report \# SOTV-DEV-ED99-076, October, 1999. 